\title{
CONSTRAINT GRADIENT PROJECTIVE METHOD FOR STABILIZED DYNAMIC SIMULATION OF CONSTRAINED MULTIBODY SYSTEMS
}

\author{
Zdravko Terze \\ University of Zagreb \\ F. Mech. Eng. Naval Arch. \\ Department of Aerospace Engineering
}

\author{
Joris Naudet \\ Vrije Universiteit Brussel \\ Department of Mechanical \\ Engineering
}

\author{
Dirk Lefeber \\ Vrije Universiteit Brussel \\ Department of Mechanical \\ Engineering
}

\begin{abstract}
Constraint gradient projective method for stabilization of constraint violation during integration of constrained multibody systems is in the focus of the paper. Different mathematical models for constrained MBS dynamic simulation on manifolds are surveyed and violation of kinematical constraints is discussed. As an extension of the previous work focused on the integration procedures of the holonomic systems, the constraint gradient projective method for generally constrained mechanical systems is discussed. By adopting differentialgeometric point of view, the geometric and stabilization issues of the method are addressed. It is shown that the method can be applied for stabilization of holonomic and non-holonomic constraints in Pfaffian and general form.
\end{abstract}

\section{INTRODUCTION}

During dynamical simulation of constrained multibody systems, a violation of system kinematical constraints is the basic source of time-integration errors and frequent difficulty that analyst have to cope with. As will be surveyed in the following chapters, if the governing equations are not turned into so called minimal form, but dynamic simulation is based on the mathematical models expressed via redundant coordinates, a constraint violation stabilization method have to be applied during integration procedure. Baumgarte stabilization method that minimizes violations can be applied for this purpose, but this algorithm is dependent on empirical feedback gains and has some limitations [1]. Different methods that provide full stabilization of system constraints are discussed in $[2,3,4]$.

The stabilized integration procedure, whose stabilization step is based on projection of the integration results to the underlying constraint manifold via post-integration correction of selected coordinates, is proposed and compared with similar integration schemes in [5]. The integration procedure is compatible with many ODE integrators and provides full stabilization of system constraint violation, but its utilization is confined to the holonomic systems only. As an extension of the previous work, a further elaboration of the projective stabilization step described in [5] is reported in this paper. Based on the gained insight, the geometric and stabilization properties of the projection algorithm are addressed when routine is applied for stabilization of holonomic and nonholonomic constraints in Pfaffian and general form. In the case of holonomic systems it is shown that, once the subvector is optimally partitioned at the position level, it can be used automatically for stabilization at the velocity level as well. The next question is: would it be possible to apply the proposed algorithm in the framework of simulation procedures of nonholonomic systems ? It is shown that in the case of nonholonomic systems, the optimally partitioned subvectors can generally have a different structure for 'positions' and velocities.

\section{UNCONSTRAINED MBS ON MANIFOLDS}

Unconstrained multibody system (MBS) is an autonomous Lagrangian system. If $n \mathrm{DOF}$ is assumed, the system evolution in configuration space $\mathrm{R}^{n}$ is described (by definition) by Lagrangian equations [6]

$$
\frac{\mathrm{d}}{\mathrm{d} t}\left(\frac{\partial L}{\partial \dot{\mathbf{x}}}\right)-\frac{\partial L}{\partial \mathbf{x}}=\Gamma^{*}, \mathbf{M}(\mathbf{x}) \ddot{\mathbf{x}}=\mathbf{Q}^{*}(\mathbf{x}, \dot{\mathbf{x}}, t) .
$$


By taking differentiable manifold approach, the configuration space $\mathrm{R}^{n}$ is considered to be a manifold $\mathrm{M}^{n}$ covered by coordinate system $\mathbf{x}(t)$ (in mathematical jargon of modern differential geometry: locally covered by chart $\mathbf{x}$ ). The solution of (1) is a dynamical trajectory $\mathrm{T}: x^{i}=x^{i}(t)$ of the system in $n$-dimensional manifold of configuration $\mathrm{M}^{n}$.

With every point on manifold of configuration, $\quad \mathbf{x} \in \mathrm{M}$, the $n$ dimensional tangent space $T_{\mathbf{x}} \mathrm{M}$ is affiliated, where system virtual displacements $\delta \mathbf{x}$ and velocities $\dot{\mathbf{x}}$ are contained, $\delta \mathbf{x} \in T_{\mathbf{x}} \mathrm{M}, \mathrm{d} \mathbf{x} \in T_{\mathbf{x}} \mathrm{M}, \dot{\mathbf{x}} \in T_{\mathbf{x}} \mathrm{M}$. The manifold $\mathrm{M}$ and the union of all tangent spaces at the various points $\mathbf{x}$ make another, $2 n$ dimensional, manifold called tangent bundle, $T \mathrm{M}: \bigcup_{\mathbf{x} \in \mathrm{M}^{\mathrm{n}}} T_{\mathbf{x}} \mathrm{M}$, covered by the coordinates $\mathbf{x}, \dot{\mathbf{x}}: T \mathrm{M}=\left\{(\mathbf{x}, \dot{\mathbf{x}}): \mathbf{x} \in \mathrm{M}, \dot{\mathbf{x}} \in T_{\mathbf{x}} \mathrm{M}\right\}$ [7] (being mathematically not very rigorous, tangent bundle can be observed as a velocity phase space known from 'traditional' approach).

Manifold $\mathrm{M}$ is not a vector space. By adopting system generalized mass matrix $\mathbf{M}(\mathbf{x})$ (positive definite) as a Riemannian metric on the manifold of configuration [8], a scalar product in the each tangent space $T_{\mathbf{x}} \mathrm{M}$ is given by $\langle\mathbf{y}, \mathbf{z}\rangle_{\mathbf{M}(\mathbf{x})}=\mathbf{y}^{\mathrm{T}} \mathbf{M}(\mathbf{x}) \mathbf{z}, \mathbf{y}, \mathbf{z} \in T_{\mathbf{x}} \mathbf{M}$ [9]. Now, with the metric so defined, the tangent space $T_{\mathbf{x}} \mathrm{M}$ ('the fiber of the tangent bundle at point $\mathbf{x}^{\prime}$ ) becomes a local Euclidean vector space spanned by covariant basis $\hat{\mathbf{g}}_{\mathbf{x}_{i}}, g_{i j}=\hat{\mathbf{g}}_{\mathbf{x}_{i}} \cdot \hat{\mathbf{g}}_{\mathbf{x}_{j}}$. By introducing a reciprocal contravariant basis $\hat{\mathbf{g}}_{\mathbf{x}}^{i}$ [10], the vectors in tangent spaces can be expressed using their contravariant and covariant representations $\hat{\dot{\mathbf{x}}}=\dot{x}^{i} \hat{\mathbf{g}}_{\mathbf{x}_{i}}$, $\dot{\mathbf{x}}=\left[\dot{x}^{i}\right], \quad \hat{\dot{\mathbf{x}}}=\dot{x}_{i} \hat{\mathbf{g}}_{\mathbf{x}}^{i}, \quad \dot{\mathbf{x}}^{*}=\left[\dot{x}_{i}\right], \quad \mathbf{G}_{\mathbf{x}}=\left[\hat{\mathbf{g}}_{\mathbf{x}_{1}}, \ldots ., \hat{\mathbf{g}}_{\mathbf{x}_{n}}\right]^{\Gamma}$ The infinitesimal distance between two points on manifold (the system kinematical line element) is defined (scalar product in manifold tangent space) by $\mathrm{d} s^{2}=g_{i j} \mathrm{~d} x^{i} \mathrm{~d} x^{j}$, $\mathbf{M}(\mathbf{x})=\left\lfloor g_{i j}\right\rfloor$.

Being dependent both on $\mathbf{x}$ and $\dot{\mathbf{x}}$, the system kinetic energy $E k(\mathbf{x}, \dot{\mathbf{x}}): T \mathrm{M}^{n} \rightarrow \mathrm{R}$ is defined on tangent bundle $T \mathrm{M}^{n}$. It is a quadratic, positive definite form on the each tangent space

$$
E k=\frac{1}{2}\|\dot{\mathbf{x}}\|_{\mathbf{M}(\mathbf{x})}^{2}=\frac{1}{2} \dot{\mathbf{x}}^{\mathrm{T}} \mathbf{M}(\mathbf{x}) \dot{\mathbf{x}}, \dot{\mathbf{x}} \in T_{\mathbf{x}} \mathrm{M} .
$$

By following a standard procedure, Lagrangian equations (1) can be turned into the $2 n$ ODE form

$$
\begin{aligned}
& \dot{\mathbf{v}}=\mathbf{M}^{-1}(\mathbf{x}) \mathbf{Q}^{*}(\mathbf{x}, \mathbf{v}, t), \quad \dot{\mathbf{x}}=\mathbf{v}, \quad \overline{\mathbf{x}}=\left[\begin{array}{l}
\mathbf{x} \\
\mathbf{v}
\end{array}\right], \\
& \dot{\overline{\mathbf{x}}}=\mathrm{f}(\overline{\mathbf{x}}), \quad \mathrm{f}(\overline{\mathbf{x}})=\left[\begin{array}{c}
\mathbf{v} \\
\mathbf{M}^{-1} \mathbf{Q}^{*}
\end{array}\right],
\end{aligned}
$$

which solution is the integral curve of the vector field $\mathrm{f}(\overline{\mathbf{x}})$ on tangent bundle ('velocity phase space') for a set of Cauchy data $\left(t_{0}, \overline{\mathbf{x}}_{\mathbf{0}}\right)$.

\section{GEOMETRIC PROPERTIES OF CONSTRAINTS 3.1. Holonomic constraints}

Holonomic constraints

$$
\Phi(\mathbf{x}, t)=\mathbf{0}, \boldsymbol{\Phi}(\mathbf{x}, t): \mathrm{R}^{n} \times \mathrm{R} \rightarrow \mathrm{R}^{r}
$$

that are imposed on the system restrict system configuration space ('positions'): a trajectory $\mathrm{T}: x^{i}=x^{i}(t)$ 'moves' on the $n-r$ dimensional constraint manifold $\mathrm{S}^{n-r}(t)$, $\mathrm{S}^{n-r}(t)=\{\mathbf{x} \in \mathrm{M}, \Phi(\mathbf{x}, t)=\mathbf{0}\}, t \geq 0, \mathbf{x}\left(t_{0}\right) \in \mathrm{S}^{n-r}\left(t_{0}\right)$, at the velocity level they induce constraint equation

$$
\Phi^{*}{ }_{\mathbf{x}}(\mathbf{x}, t) \dot{\mathbf{x}}=-\Phi_{t}=\boldsymbol{\tau}
$$

that is linear in velocities. If constraints are scleronomic (constraints do not depend on time explicitly, i.e. $\Phi(\mathbf{x})=\mathbf{0}$ ), the constraints at velocities take a form $\Phi^{*}{ }_{\mathbf{x}}(\mathbf{x}) \dot{\mathbf{x}}=\mathbf{0}$, which determine $\hat{\dot{\mathbf{x}}}$ as a tangent to the position constraint manifold, $\operatorname{rank}\left(\Phi^{*}{ }_{\mathbf{x}}=\frac{\partial \Phi(\mathbf{x})}{\partial \mathbf{x}}\right)=r$

The system is said to be a holonomic one and posses $n-r$ degrees of freedom (DOF).

The constraint matrix $\Phi_{\mathbf{x}}^{*}(\mathbf{x}, t)$ can be written in the form $\Phi_{\mathbf{x}}^{* \mathrm{~T}}(\mathbf{x}, t)=\left[\varphi_{1}^{*}, \ldots, \varphi_{r}^{*}\right], \quad \varphi_{i}^{*}=\left[\varphi_{i}\right], \quad \hat{\varphi}_{1}=\varphi_{i} \hat{\mathbf{g}}_{\mathbf{x}}^{i}$. The vectors $\hat{\varphi}_{1}, \ldots, \hat{\varphi}_{r}$ represent gradients to the constraint hypersurfaces, determined in the configuration space by the equations $\Phi(\mathbf{x}, t)=\mathbf{0}$, i.e. $\quad \hat{\varphi}_{1}=\left[\operatorname{grad} \Phi_{1}=0\right], \ldots$, $\hat{\varphi}_{r}=\left[\operatorname{grad} \Phi_{\mathrm{r}}=0\right]$.

The vectors $\varphi_{i}^{*}$ are linearly independent and span $r$ dimensional constraint subspace $\mathbf{C}_{\mathbf{x}}^{r} \quad$ [11]. Kinematically admissible virtual displacements $\delta \mathbf{x}$ are restricted to the $n-r$ dimensional tangent space $T_{\mathbf{x}} \mathbf{S}^{n-r}$ that is orthogonal to $\mathbf{C}_{\mathbf{x}}^{r}$. Together, subspaces $\mathbf{C}_{\mathbf{x}}^{r}$ and $T_{\mathbf{x}} \mathbf{S}^{r}$ span fiber of tangent bundle of unconstrained system $T_{\mathbf{x}} \mathrm{M}^{n}$ (tangent space) at point $\mathbf{x}: \quad T_{\mathbf{x}} \mathbf{S}^{n-r} \cap \mathbf{C}_{\mathbf{x}}^{r}=0, \quad T_{\mathbf{x}} \mathbf{S}^{n-r} \cup \mathbf{C}_{\mathbf{x}}^{r}=T_{\mathbf{x}} \mathbf{M}^{n} . \quad$ Thus, 
orthogonal-complement matrix $\mathbf{R}(\mathbf{x}, t), \mathbf{R}(\mathbf{x}, t)=\left\lfloor\mathbf{r}_{1}, \ldots . . \mathbf{r}_{n-r}\right\rfloor$, that satisfy complementarity equation $\Phi^{*}{ }_{\mathbf{x}}(\mathbf{x}, t) \mathbf{R}(\mathbf{x}, t)=\mathbf{0}$, can be determined where $\hat{\mathbf{r}}_{1}, \ldots . ., \hat{\mathbf{r}}_{n-r}$ are basis vectors of $T_{\mathbf{x}} \mathbf{S}^{n-r}[12]$.

In the case of scleronomic constraints, the system velocities (not just system virtual displacements $\delta \mathbf{x}$ ) are entirely contained in $T_{\mathbf{x}} \mathrm{S}^{n-r}$ and can be expressed with respect to the basis $\mathbf{G}_{\mathbf{r}}=\left[\hat{\mathbf{r}}_{1}, \ldots . ., \hat{\mathbf{r}}_{n-r}\right]^{\mathrm{T}}$ only (instead of representation $\hat{\dot{\mathbf{x}}}=\dot{\mathbf{x}}^{\mathrm{T}} \mathbf{G}_{\mathbf{x}}$ that is expressed via basis $\mathbf{G}_{\mathbf{x}}$ that 'covers' whole unconstrained tangent space $T_{\mathbf{x}} \mathrm{M}^{n}$ ), i.e.

$$
\hat{\dot{\mathbf{x}}}=\dot{\mathbf{z}}^{\mathrm{T}} \mathbf{G}_{\mathbf{r}}, \dot{\mathbf{x}}=\mathbf{R} \dot{\mathbf{z}} \text {. }
$$

In the equation (5) system velocities are represented via independent generalized velocities $\dot{\mathbf{z}}$ (virtual speeds [11]).

If the constraints are rheonomic (constraints do depend explicitly on time, a constraint manifold $\mathbf{S}^{n-r}$ 'moves' within $\mathrm{M}^{n}$ ), the velocities are not totally sunk in $T_{\mathbf{x}} \mathrm{S}^{n-r}$ and can be expressed via $T_{\mathbf{x}} \mathrm{S}^{n-r}$ basis and additional vector $\boldsymbol{\alpha}(\mathbf{x}, t)$ (7) due to the time dependency of $\mathbf{S}^{n-r}$. The time derivative of (7) yields (8) by means of which accelerations are constrained

$$
\begin{aligned}
& \dot{\mathbf{x}}=\mathbf{R} \dot{\mathbf{z}}+\boldsymbol{\alpha}(\mathbf{x}, t) \\
& \ddot{\mathbf{x}}=\mathbf{R} \ddot{\mathbf{z}}+\dot{\mathbf{R}} \dot{\mathbf{z}}+\dot{\alpha}(\mathbf{x}, t)
\end{aligned}
$$

\subsection{Non-holonomic constraints}

If, beside $h$ holonomic constraints (3), the additional $n h$ non-holonomic constraints

$$
\Psi(\mathbf{x}, \dot{\mathbf{x}}, t)=\mathbf{0}
$$

are imposed on the system:

a) they do not restrict system configuration space (system constraint manifold $\mathrm{S}^{n-r}$ maintains the same dimension, $r=h)$

b) they impose additional velocity constraints on holonomic constraint manifold tangent bundle TS , $\dot{\mathbf{x}} \in T_{\mathbf{x}}^{n-r-n h} \mathbf{S}^{n-r} \subset T_{\mathbf{x}}^{n-r} \mathbf{S}^{n-r}$.

If non-holonomic constraints are linear in velocities, i.e. can be given in Pfaffian form

$$
\Psi=\mathbf{B}^{*}(\mathbf{x}, t) \dot{\mathbf{x}}-\beta(\mathbf{x}, t)=\mathbf{0},
$$

the system constraint equations can be written as follows:

$$
\begin{aligned}
& {\left[\begin{array}{l}
\Phi_{\mathbf{x}}^{*}(\mathbf{x}, t) \\
\mathbf{B}^{*}(\mathbf{x}, t)
\end{array}\right] \dot{\mathbf{x}}=\left[\begin{array}{l}
\tau \\
\boldsymbol{\beta}
\end{array}\right]} \\
& {\left[\begin{array}{l}
\Phi_{\mathbf{x}}^{*}(\mathbf{x}, t) \\
\mathbf{B}^{*}(\mathbf{x}, t)
\end{array}\right]=\Phi_{\mathbf{x} n h}^{*}, \Phi_{\mathbf{x} n h}^{*} \in \mathrm{R}^{h+n h \times n} .}
\end{aligned}
$$

As it was the case with systems that posses only holonomic constraints, the orthogonal-complement matrix $\mathbf{R}_{n h}$ that satisfy complementarity equation $\Phi_{\mathbf{x} n h}^{*} \mathbf{R}_{n h}=\mathbf{0}$ can be determined via numerical methods described in literature [12, $13,14]$.

\section{MATHEMATICAL MODELS OF CMS}

\subsection{Models with inherent constraint violation problem}

To avoid high-index DAE formulations, mathematical model for dynamic simulation of multibody systems with imposed holonomic constraints (3) is often shaped as a differential-algebraic system (DAE) of index 1 (redundant coordinates formulation, ideal constraints' reaction forces are assumed) $[1,16]$

$$
\left[\begin{array}{cc}
\mathbf{M} & \boldsymbol{\Phi}^{* \mathrm{~T}} \\
\boldsymbol{\Phi}^{*}{ }_{\mathrm{x}} & \mathbf{0}
\end{array}\right]\left[\begin{array}{l}
\ddot{\mathbf{x}} \\
\mathbf{\lambda}
\end{array}\right]=\left[\begin{array}{c}
\mathbf{Q}^{*} \\
\xi
\end{array}\right],
$$

where Lagrangian equations of the first type

$$
\begin{aligned}
& \mathbf{M}(\mathbf{x}) \ddot{\mathbf{x}}+\Phi_{\mathbf{x}}^{* \mathrm{~T}}(\mathbf{x}, t) \mathbf{\lambda}=\mathbf{Q}^{*}(\dot{\mathbf{x}}, \mathbf{x}, t), \\
& \mathbf{x} \in \mathrm{R}^{n}, \Phi_{\mathbf{x}}^{*} \in \mathrm{R}^{r \times n}, \operatorname{rank}\left[\Phi_{\mathbf{x}}^{*}\right]=r
\end{aligned}
$$

and the constraint equations at the acceleration level (time derivative of (4))

$$
\Phi_{\mathbf{x}}^{*}(\mathbf{x}, t) \ddot{\mathbf{x}}=\xi,
$$

are put together. System (13) is uniquely solvable for the set of consistent initial values. It can be integrated in time to obtain kinetic motion of the system as well as constraint reactions. Although constraints at the acceleration level will be immanently satisfied since (15) is included in mathematical model (13) and will be explicitly solved during integration, the numerical non-stability of (15) can induce constraints violation at the both position and velocity level [9].

If the constraint reactions are not of interest, by explicit elimination of $\boldsymbol{\lambda}$ [17] DAE can be transformed to $2 n$ ODE, $\dot{\overline{\mathbf{x}}}=\mathbf{w}(\overline{\mathbf{x}}), \quad \overline{\mathbf{x}}=\left[\mathbf{x}^{\mathrm{T}} \mathbf{v}^{\mathrm{T}}\right]^{\mathrm{T}}$, whose solution determine time evolution of the system. Although analytical solution curves for consistent initial conditions will move on tangent 
bundle $T^{n-r} \mathrm{~S}^{n-r}$ (having satisfied all constraints imposed on the system), a numerical solution will tend to drift away from constraint manifold.

DAE system (13) can be transformed to ODE also by projecting dynamical equations (14) to the local tangent space $T_{\mathbf{x}} \mathrm{S}^{n-r}$, thus eliminating system constraint reactions:

$$
\mathbf{R}(\mathbf{x}, t)^{\mathrm{T}} \mathbf{M}(\mathbf{x}) \ddot{\mathbf{x}}=\mathbf{R}(\mathbf{x}, t)^{\mathrm{T}} \mathbf{Q}^{*}(\dot{\mathbf{x}}, \mathbf{x}, t)
$$

By adding acceleration equation (15), the governing equations can be put in the form of $\mathrm{n}$ ODE of 2. order [18]

$$
\left[\begin{array}{c}
\mathbf{R}^{\mathrm{T}} \mathbf{M} \\
\boldsymbol{\Phi}^{*}{ }_{\mathbf{x}}
\end{array}\right] \ddot{\mathbf{x}}=\left[\begin{array}{c}
\mathbf{R}^{\mathrm{T}} \mathbf{Q}^{*} \\
\boldsymbol{\xi}
\end{array}\right] .
$$

As it was case with (13), during straightforward integration of (17) the constraints might be violated at the both position and velocity level.

If dynamical model is shaped by employing a set of independent velocities $\dot{\mathbf{Z}}$ (re-shaping (16) by using (7) and (8) where $\alpha$ disappears in the case of scleronomic constraints), the governing equations become $n-r$ ODE system [3, 19]

$$
\mathbf{R}^{\mathrm{T}} \mathbf{M} \mathbf{R} \ddot{\mathbf{z}}=\mathbf{R}^{\mathrm{T}} \mathbf{Q}-\mathbf{R}^{\mathrm{T}} \mathbf{M}(\dot{\mathbf{R}} \dot{\mathbf{z}}+\dot{\alpha})
$$

Although ODE system (18) has the same dimension as system modelled in minimal form (19), there is a big difference in geometry of coordinate representations, which strongly influence utilization characteristics of the two formulations.

The configuration coordinates that correspond to the generalized velocities $\dot{\mathbf{z}}$ generally do not have 'physical' meaning since they, except for the special cases, do not parameterize constraint manifold $\mathrm{S}^{n-r}$. Therefore, introduction of a set of independent velocities $\dot{\mathbf{z}}$ assures no constraint violation at the velocity level, while the constraints at the configuration ('positions') level are still prone to integration errors.

If, beside holonomic constrains, the additional nonholonomic constraints given in Pfaffian form (10) are imposed on the system, the procedures $[9,11]$, similar to those described above, allow for shaping of mathematical models given by (13), (17), (18).

\subsection{Minimal form formulation}

The governing equations of holonomic CMS can be turn into the minimal form of $n-r$ ODE (the equations of motion) that are not prone to constraint violations of any kind only if, based on $\Phi(\mathbf{x}, t)=\mathbf{0}$, a new set of configuration coordinates $\mathbf{y}(t) \in \mathrm{R}^{n-r}$ that describe constraint manifold can be established $\mathrm{S}^{n-r}(t)$ (thus determining system configuration). In this case, equations of motion can be shaped in the minimal form $[11,15]$

$$
\mathbf{M}_{\mathbf{y}}(\mathbf{y}, t) \ddot{\mathbf{y}}=\mathbf{Q}_{\mathbf{y}}^{*}(\mathbf{y}, \dot{\mathbf{y}}, t) .
$$

Since $\mathbf{y}(t) \in \mathrm{R}^{n-r}$ so defined parameterize $\mathrm{S}^{n-r}(t)$, the numerical integration errors do not produce system constraint violation of any kind (but do affect system kinetic motion, however).

\section{CONSTRAINT GRADIENT PROJECTIVE METHOD FOR STABILIZATION OF CONSTRAINT VIOLATION}

If system governing equations are not turned into minimal form (19) but dynamic simulation is based on the mathematical models (13), (17), or (18) and whole vector of system redundant coordinates is being integrated, a constraint violation stabilization method have to be applied during integration procedure. Another, well known, algorithm for integration of redundant systems is the coordinates partitioning procedure [20].

The stabilization algorithm proposed in [5] is based on the projection of the step-post-integration results (during integration via redundant coordinates, constraint violation is likely to occur) to the constraint manifold in the course of simulation.

The starting point of the simulation procedure is mathematical model in form (17). After integration phase, the projective stabilization step at the position level is accomplished by correction of the dependent coordinates subvector $\mathbf{x}^{\mathrm{d}}$, providing thus the shifting of system state-point $\mathbf{x}$ back to the constraint manifold $\mathrm{S}^{n-r}$ (iterative solving of (3) that brings $\mathbf{x}$ in accordance with (3) up to the required accuracy, $\mathbf{x}^{\mathrm{d}}$ is to be treated as unknown variable). The procedure is then repeated at the velocity level by correcting $\dot{\mathbf{x}}^{\mathrm{d}}$ to bring $\dot{\mathbf{x}}$ in accordance with (4). As a stabilization step final result, the time-integration values $\dot{\mathbf{x}}, \mathbf{x}$ are projected to the constraint manifold tangent bundle $T \mathrm{~S}$ satisfying thus constraints of the system fully. As will be seen later, a crucial point of the algorithm is appropriate selection of sub-vectors $\mathbf{x}^{\mathrm{d}}$ and $\dot{\mathbf{x}}^{\mathrm{d}}$ to provide the optimal stabilization effect of the procedure.

Criteria for the coordinates selection can be expressed geometrically: basically, every selection that returns subvector of dependent coordinates $\mathbf{x}^{\mathrm{d}}$ which basis vectors have nonzero projections on the constraint subspace $\mathbf{C}_{\mathbf{x}}^{r}$ (the corresponding $r \times r$ submatrix of constraint matrix $\Phi_{\mathbf{x}}^{*}$ is non-singular) is correct one and can be used for stabilization procedure. Consequently, the basis vectors of variables $\mathbf{x}^{\mathrm{i}}$ have projections on tangent space of constraint manifold $T_{\mathbf{x}} \mathrm{S}^{\mathrm{n}-\mathrm{r}}$ that is complement to $\mathbf{C}_{\mathbf{x}}^{r}$. If the extracted subvectors do not satisfy specified conditions, the selection is not a valid one and the calculation will fail. Technically, if the system is holonomic and constrained on manifold $\mathrm{S}^{n-r}$, the coordinate selection can be performed via pivoting operations on the constraint matrix $\Phi_{\mathbf{x}}^{*}, \operatorname{rank}\left(\Phi_{\mathbf{x}}^{*}\right)=r$, by means of which 
the subvectors of dependent and independent coordinates $\mathbf{x}^{\mathrm{d}} \in \mathrm{R}^{r}$ and $\mathbf{x}^{\mathrm{i}} \in \mathrm{R}^{n-r}$ is extracted (coordinate partitioning algorithm). For the purpose of optimal selection, a projective criterion to the coordinate partitioning method can be utilized [21].

\subsection{Integration errors along manifold}

The main problem that may occur during stabilization procedure is an inadequate coordinate selection that may have a negative effect on the integration accuracy along the constraint manifold. Although, as it was explained, every partitioning that returns acceptable subvectors can be used for the stabilization projective step, a non-optimal choice of the coordinate subvectors may cause an increase of the numerical errors along the manifold during stabilization part of the integration procedure (numerical errors along constraint manifold affect system evolution in time i.e. its kinetic motion). If this happens, a correction of the constraint violation will be accomplished at the expense of the 'kinetic motion' accuracy obtained by the system variables $\dot{\mathbf{x}}, \mathbf{x}$ ODE integrators.

\subsubsection{Stabilization of the system configuration constraints}

The 'mechanism' of emerging of the numerical errors along configuration manifold, because of an inadequate partitioning during the stabilization procedure of holonomic systems, is outlined in Fig. 1, where an illustrative example $\mathbf{x} \in \mathrm{R}^{2}, \mathrm{~S}^{1}$ is discussed [22].

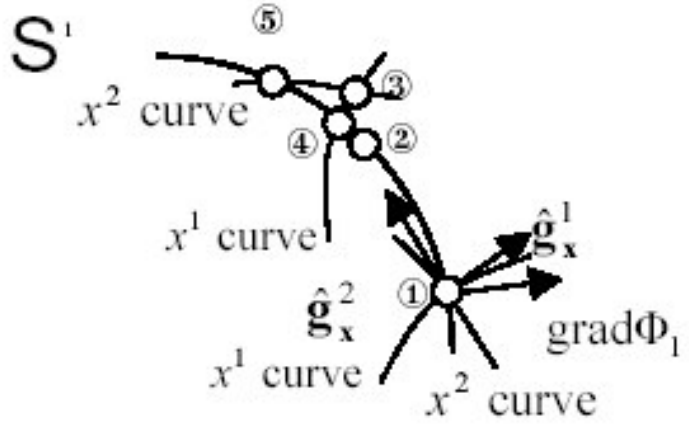

Fig. 1: Correction of the configuration constraint violation

Assuming that, starting from (1), an integration of ODE gives result (3) instead of exact position (2), a projection on the constraint manifold $\mathrm{S}^{1}$ by adjusting coordinate $x^{1}$ (solving 'position' i.e. configuration constraint equation (3) along $x^{1}$ curve by treating $x^{1}$ as dependent i.e. unknown variable) yields result (4) that is consistent to the constraint. If instead of $x^{1}$, the variable $x^{2}$ was chosen to be a dependent coordinate, an adjustment of the integration result along $x^{2}$ curve would yield solution 5 , which is also consistent to the constraint but contains considerable error along the manifold $\mathrm{S}^{1}$.

A remedy for the problem of an inadequate selection of dependent coordinates has been offered in [21], where a projective criterion to the coordinate partitioning method is introduced. For a given set of coordinates of unconstrained system, the criterion allows for the optimal choice of dependent/independent coordinates which, consequently, gives opportunity to minimize integration error along manifold.

The main idea is to determine those $r$ coordinates which direction vectors $\hat{\mathbf{g}}_{\mathbf{x}}^{i}$ deliver the biggest relative projections to the $\mathbf{C}_{\mathbf{x}}^{r}$ and select them as dependent variables which will be adjusted during the stabilization procedure. By correcting the coordinates whose direction vectors align well with the constraint gradients (that point directions toward constraint surfaces and span $\mathbf{C}_{\mathbf{x}}^{r}$ ), it is ensured that the correction procedure will shift a state-point of the system 'as direct as possible' to the constraint hypersurfaces, minimizing thus an error along constraint manifold. Along this line, in the example shown in Fig. 1, the variable $x^{1}$ is chosen to be a dependent coordinate since its basis vector $\hat{\mathbf{g}}_{\mathbf{x}}^{1}$ delivers a big projection on $\mathbf{C}_{\mathbf{x}}^{r}=\operatorname{grad}\left[\Phi_{1}=0\right]$ (in this illustrative example the constraint subspace $\mathbf{C}_{\mathbf{x}}^{r}$ is one-dimensional, spanned by $\operatorname{grad}\left[\Phi_{1}=0\right]$ ).

Generally, the stabilization projective algorithm [5] that utilizes gradient criterion as outlined above is similar to the algorithms known from the optimization procedures, where constraint gradients are determined within the framework of optimization algorithms in order to detect the 'fastest' directions toward constraint surfaces [23].

Note: if the system illustrated in Fig. 1 was modeled in minimal form (19), the formulation would yield a mathematical model with a single coordinate; the coordinate direction vector would be orthogonal in every moment to $\operatorname{grad}\left[\Phi_{1}(\mathbf{x})=0\right]$ and fully projected to the one-dimensional tangent space of the constraint manifold $\mathrm{S}^{1}$.

\subsubsection{Stabilization of the velocity constraints}

The projective criterion for coordinate selection can be applied for minimization of the numerical errors in the process of correction of constraint violation at the velocity level as well. Here, an application of the criterion enhances a definiteness of the velocity constraints algebraic system (4), providing thus a better numerical accuracy of the stabilization procedure. This feature is illustrated by an example $\mathbf{x} \in \mathrm{R}^{3}$, $S^{1}=\left\{\mathbf{x} \in R^{3}, \Phi_{1}(\mathbf{x})=0\right\}$, shown in Fig. 2. Because of simplicity, scleronomic system and orthogonal basis $\hat{\mathbf{g}}_{\mathbf{x}}^{1}, \hat{\mathbf{g}}_{\mathbf{x}}^{2}, \hat{\mathbf{g}}_{\mathbf{x}}^{3}$ are assumed.

If the velocity equation (4) is written in the 'vectorial' form, for the analysed case it reads

$$
\operatorname{grad} \Phi_{1} \cdot \hat{\dot{\mathbf{x}}}=0 .
$$


In (20), the components of $\operatorname{grad} \Phi_{1}$ represent coefficients of the linear algebraic system that, for a general mathematical model, is given by (4). By applying the projective criterion and choosing $\dot{x}^{3}$, which direction vector $\hat{\mathbf{g}}_{\mathbf{x}}^{3}$ (in this 'academic' illustrative situation) is almost collinear to $\operatorname{grad} \Phi_{1}$, as a dependent coordinate, the potential numerical errors in independent coordinates $\dot{x}^{1}$ and $\dot{x}^{2}$ would not affect considerably the solution $\dot{x}^{3} \approx 0$ of the velocity constraint equation (20). This is because of the small magnitudes of the coordinates of $\operatorname{grad} \Phi_{1}$ along the basis vectors $\hat{\mathbf{g}}_{\mathbf{x}}^{1}$ and $\hat{\mathbf{g}}_{\mathbf{x}}^{2}$ (small projections of $\operatorname{grad} \Phi_{1}$ on $\hat{\mathbf{g}}_{\mathbf{x}}^{1}$ and $\hat{\mathbf{g}}_{\mathbf{x}}^{2}$ ) that multiply $\dot{x}^{1}$ and $\dot{x}^{2}$ while solving (20) for $\dot{x}^{3}$.

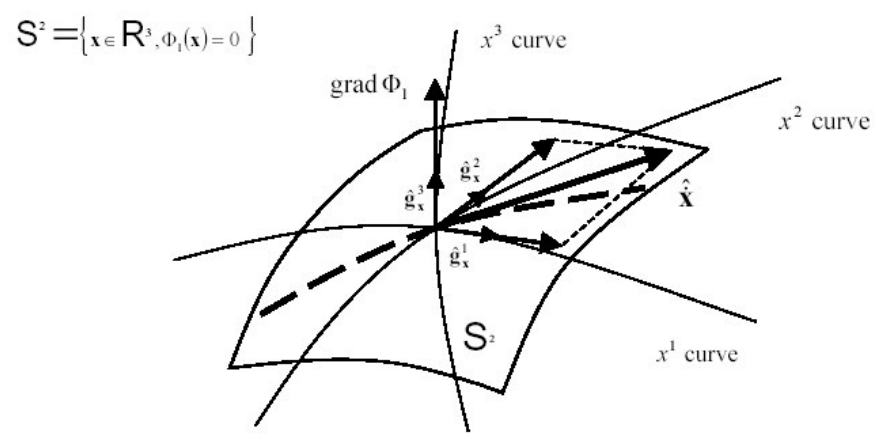

Figure 2:Correction of constraint violation at the velocity level

\subsection{Structure of the partitioned subvectors}

So far, constraint gradient projective method has been discussed for stabilization of constraint violation during dynamic simulation of holonomic system only [5]. Would it be possible to apply proposed algorithm in the framework of simulation procedures of non-holonomic systems? If, in the case of holonomic system simulation, partitioned subvector at the position level is selected, can the same subvector be used automatically for stabilization at the velocity level as well? Is it valid in any case?

To get answers on these questions and gain further insight into described procedure, it is illustrative to observe characteristics of the proposed algorithm at the tangent bundle $T \mathrm{M}=\left\{(\mathbf{x}, \dot{\mathbf{x}}): \mathbf{x} \in \mathrm{M}, \dot{\mathbf{x}} \in T_{\mathbf{x}} \mathrm{M}\right\}$ of an unconstrained system.

As explained, $T \mathrm{M}$ is $2 n$-dimensional Riemannian manifold with a metric $\mathbf{M}_{T M}=\operatorname{diag}(\mathbf{M}(\mathbf{x}), \mathbf{M}(\mathbf{x}))$, where a configuration of the system as well as its velocities can be studied [24]. If constraints are present, they are represented in $T \mathrm{M}$ by the configuration and velocity submanifolds, by means of which the possible states of system are determined. Observed at $T \mathrm{M}$, the constraint gradient projective method can be studied for each submanifolds separately. By using the projective criterion for both manifolds, characteristics of the partitioning procedure that for a given set of coordinates $\mathbf{x} \in \mathrm{M}, \dot{\mathbf{x}} \in T_{\mathbf{x}} \mathrm{M}$ provides the optimal dependent/independent subvectors, can be learned as follows.

\subsubsection{Holonomic constraints}

The configuration submanifold $\mathrm{S}^{n-r}$ is determined by the equation (3) i.e.

$$
\mathrm{S}^{n-r}=\{\mathbf{x} \in \mathrm{M}, \Phi(\mathbf{x}, t)=\mathbf{0}\} .
$$

The submanifold $\mathbf{V}^{n-r}$, by means of which the system velocities $\dot{\mathbf{X}}$ are constrained, is defined by (4), thus

$$
\bigvee^{n-r}=\left\{\dot{\mathbf{x}} \in T_{\mathbf{x}} \mathrm{M}, \Phi^{*}{ }_{\mathbf{x}}(\mathbf{x}, t) \dot{\mathbf{x}}=\boldsymbol{\tau}\right\} .
$$

If the constraint gradient projective method is applied for stabilization purposes at the both configuration and velocity level, the projective criterion itself is based on determination of the gradients to the constraint submanifolds $\mathrm{S}^{n-r}$ and $\mathrm{V}^{n-r}$ (as explained, this is because the extraction of the dependent coordinates of $\mathbf{x}^{\mathrm{d}}$ and $\dot{\mathbf{x}}^{\mathrm{d}}$ depend on the directions of gradients to the hypersurfaces of submanifolds $\mathrm{S}^{n-r}$ and $\mathrm{V}^{n-r}$ respectively).

Since constraint submanifold $\mathbf{S}^{n-r}$ is determined by (3), the $\mathbf{x}$ correction gradient by means of which $\mathbf{x}^{\mathrm{d}}$ is to be extracted is given by

$$
\operatorname{grad}[\mathbf{\Phi}(\mathbf{x}, t)=\mathbf{0}]=\boldsymbol{\Phi}_{\mathbf{x}}^{*}(\mathbf{x}, t) .
$$

Similarly, $\dot{\mathbf{x}}$ correction gradient, decisive for an extraction of $\dot{\mathbf{x}}^{\mathrm{d}}$ reads as

$$
\operatorname{grad}\left[\boldsymbol{\Phi}_{\mathbf{x}}^{*}(\mathbf{x}, t) \dot{\mathbf{x}}=\boldsymbol{\tau}\right]=\boldsymbol{\Phi}_{\mathbf{x}}^{*}(\mathbf{x}, t) .
$$

Now, if the expressions (23) and (24) are compared, it is obvious that the both hypersurfaces $\mathrm{S}^{n-r}$ and $\mathrm{V}^{n-r}$ have the same gradients for every point in $T \mathrm{M}$ (in fact, the both gradients depend on the current position $\mathbf{x} \in M_{\text {at }}$ the configuration manifold and $t$ only, i.e. they are independent on system velocities $\dot{\mathbf{x}}$ ). Of course, this stems from the fact that, in the case of holonomic systems, the velocity submanifold $\bigvee^{n-r}$ is determined by algebraic equations (4) (linear in $\dot{\mathbf{x}}$ !) which are, in turn, obtained by derivation of the configuration constraints (3).

Since the gradients to the both hypersurfaces $S^{n-r}$ and $\mathrm{V}^{n-r}$ are identical, it is clear that the same optimal dependent/independent subvectors at the both configuration and velocity level i.e. $\left[x_{1}, x_{2}, \ldots, x_{\mathrm{d}}\right]^{\mathrm{T}}$ and $\left[\dot{x}_{1}, \dot{x}_{2}, \ldots, \dot{x}_{\mathrm{d}}\right]^{\mathrm{T}}$ will be extracted during stabilization via constraint gradient projective method. This means that, once the partitioning procedure is performed for the configuration coordinates and subvector $\mathbf{x}^{\mathrm{d}}$ is extracted, it is not needed to be repeated at the 
velocity level (the subvector $\dot{\mathbf{x}}^{\mathrm{d}}$ of the same structure is to be chosen for the stabilization of velocities).

\subsubsection{Non-holonomic constraints}

A constraint gradient projective method can also be applied for stabilization of constraint violation of non-holonomic systems. If additional $n h$ non-holonomic constraints (9), which are imposed on the system (beside $h$ holonomic constraints (3) that define configuration manifold $\left.\mathrm{S}^{n-r}, r=h\right)$, are given in linear (Pfaffian form) (10), the submanifold $\bigvee^{n-r-n h}$ of the velocity constraints are defined by

$$
\left[\begin{array}{l}
\Phi_{\mathbf{x}}^{*}(\mathbf{x}, t) \\
\mathbf{B}^{*}(\mathbf{x}, t)
\end{array}\right] \dot{\mathbf{x}}=\Phi_{n h}^{*} \dot{\mathbf{x}}=\left[\begin{array}{l}
\tau \\
\boldsymbol{\beta}
\end{array}\right], \Phi_{\mathbf{x} n h}^{*} \in \mathrm{R}^{h+n h \times n}
$$

By considering (25), the $\dot{\mathbf{x}}$ correction gradient reads as

$$
\operatorname{grad}\left[\Phi_{n h}^{*} \dot{\mathbf{x}}=\left[\begin{array}{c}
\tau \\
\boldsymbol{\beta}
\end{array}\right]\right]=\Phi_{n h}^{*}(\mathbf{x}, t)=\left[\begin{array}{c}
\Phi_{\mathbf{x}}^{*}(\mathbf{x}, t) \\
\mathbf{B}^{*}(\mathbf{x}, t)
\end{array}\right] .
$$

Since non-holonomic constraints do not affect configuration manifold $\mathrm{S}^{n-r}$, the 'position' coordinates correction gradient is given by (23).

By comparing correction gradients (26) and (23) which do not match any more, it can be concluded that in the case of nonholonomic systems the optimal coordinates partitioning will not 'return' dependent/independent subvectors of the same structure for configuration and velocity stabilization. Beside non-equality of dimension of the subvectors $\mathbf{x}^{\mathrm{d}} \in \mathrm{R}^{r}$ and $\dot{\mathbf{x}}^{\mathrm{d}} \in \mathrm{R}^{r-n h}$, their structure will also differ in general case. Generally, in the case of non-holonomic systems, a separate partitioning procedure is necessary for stabilization at configuration and velocity level.

This is specially true if the imposed non-holonomic constraints (9) can not be put in Pfaffian form. If nonholonomic constraints are non-linear in velocities (this kind of constraints can appear as a result of certain controlling actions), it will be necessary to determine a completely new correction gradient

$$
\operatorname{grad}[\Psi(\mathbf{x}, \dot{\mathbf{x}}, t)=\mathbf{0}]=\Psi_{\dot{\mathbf{x}}},
$$

to accomplish optimal correction of the velocity constraint violation.

\section{EXAMPLE}

As an example, a snakeboard has been numerically simulated [25]. The snakeboard is modelled as a multibody system with 4 bodies connected to each other by means of pinjoints. There is one coupler, two small boards with wheels and one rotor on the coupler to model the human body. The 2 pairs of wheels cannot slide and therefore impose two non- holonomic constraints on the system. On the configuration level, the snakeboard has 6 degrees of freedom (fig. 3).

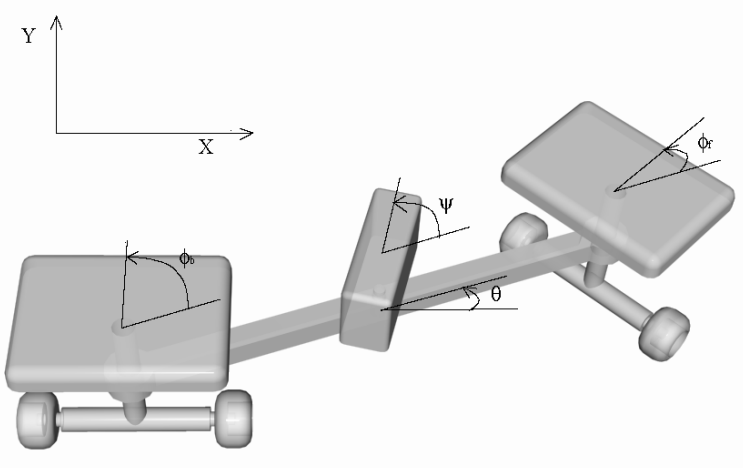

Figure 3: Snakeboard

In the simulation, following parameters were used: mass $m=6 \mathrm{~kg}$ and moment of inertia $J=0.016 \mathrm{~kg} \cdot \mathrm{m}^{2}$ of the coupler, moment of inertia $J_{w}=0.0013 \mathrm{~kg} \cdot \mathrm{m}^{2}$ of the wheel boards, moment of inertia $J_{r}=0.072 \mathrm{~kg} \cdot \mathrm{m}^{2}$ of the rotor and length $l=0.2 \mathrm{~m}$ from the centre of mass to the wheels. The initial values are $x=y=\theta=\phi_{b}=0, \psi=0.2 \mathrm{rad}$ and $\phi_{f}=\frac{\pi}{3} \mathrm{rad}$. The simulation time is $2 \mathrm{~s}$. A force $\mathbf{f}=\left(\begin{array}{ll}f_{x} & f_{y}\end{array}\right)$ of $20 \mathrm{~N}$ is acting on the centre of mass of the snakeboard, it is directed parallel to the coupler, towards the front wheels. Springs with a constant of $0.1 \mathrm{Nm} / \mathrm{rad}$ are added at the wheel boards to try to keep the initial relative angles between wheels and coupler. Another spring with a constant of $1 \mathrm{Nm} / \mathrm{rad}$ has also been added between the coupler and the rotor. The equations of motion for the system are:

$$
\begin{aligned}
& m \ddot{x}-\lambda_{1} \sin \left(\phi_{b}+\theta\right)-\lambda_{2} \sin \left(\phi_{f}+\theta\right)=f_{x} \\
& m \ddot{y}+\lambda_{1} \cos \left(\phi_{b}+\theta\right)+\lambda_{2} \sin \left(\phi_{f}+\theta\right)=f_{y} \\
& \left(J+J_{r}+2 J_{w}\right) \ddot{\theta}+J_{r} \ddot{\psi}+J_{w} \ddot{\phi}_{b}+J_{w} \ddot{\phi}_{f} \\
& -\lambda_{1} l \cos \phi_{b}+\lambda_{2} l \cos \phi_{f}=0 \\
& J_{r} \ddot{\psi}+J_{r} \ddot{\theta}=0 \\
& J_{w} \ddot{\phi}_{b}+J_{w} \ddot{\theta}=0 \\
& J_{w} \ddot{\phi}_{f}+J_{w} \ddot{\theta}=0
\end{aligned}
$$

Together with the nonholonomic constraint equations:

$$
\begin{aligned}
& -\sin \left(\phi_{b}+\theta\right) \dot{x}+\cos \left(\phi_{b}+\theta\right) \dot{y}-l \cos \left(\phi_{b}\right) \dot{\theta}=0 \\
& -\sin \left(\phi_{f}+\theta\right) \dot{x}+\cos \left(\phi_{f}+\theta\right) \dot{y}+l \cos \left(\phi_{f}\right) \dot{\theta}=0
\end{aligned}
$$

The simulation has been run under 4 different numerical integrations. First, an explicit Runge-Kutta $(4,5)$ formula with variable stepsize was used, to obtain a reference simulation S1 (fig. 4). The absolute and relative tolerances were set to $1 \mathrm{e}-13$. To test the stabilisation procedures, the equations of motion 
have subsequently be numerically integrated without stabilisation (S2), with stabilisation using the optimal choice of independent coordinates (S3) and with stabilisation using an other possible choice of independent coordinates (S4). For these simulations, a fourth order Runge-Kutta integration scheme was used with a fixed stepsize of 0.01 seconds.

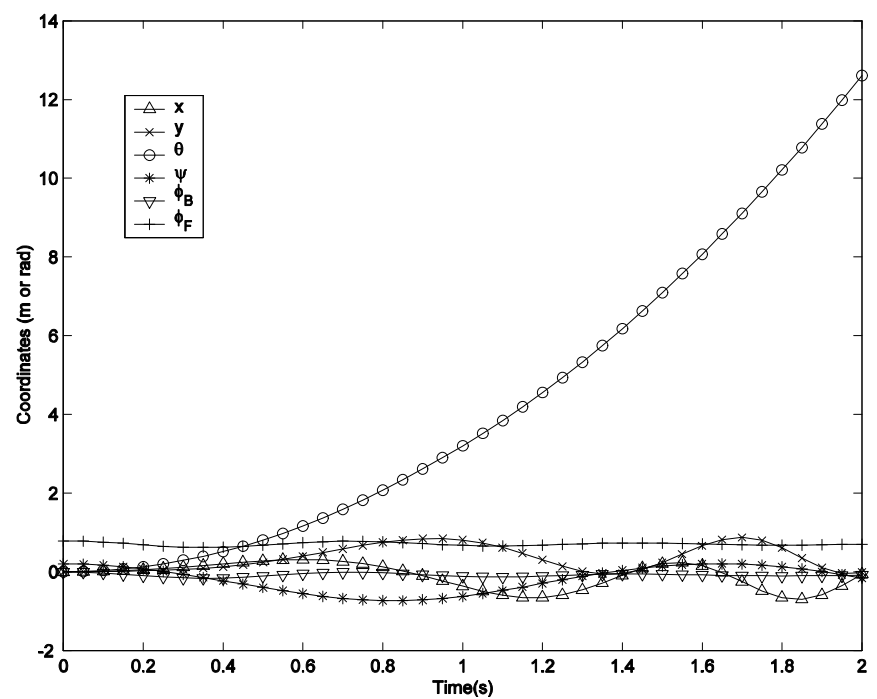

Figure 4: Evolution of the coordinates in time

The velocities $\dot{\psi}, \dot{\phi}_{b}$ and $\dot{\phi}_{f}$ do not appear in the two constraint equations and are therefore independent. The fourth independent coordinate for simulation S3 was chosen amongst $\dot{x}, \dot{y}$ and $\dot{\theta}$ using the projective criterion. The squared relative projections $d$ of the direction vectors on the tangent subspace are shown on figure 5. For simulation S3, the biggest projection was used to choose the independent velocity, it was alternatively $\dot{x}$ and $\dot{y}$.

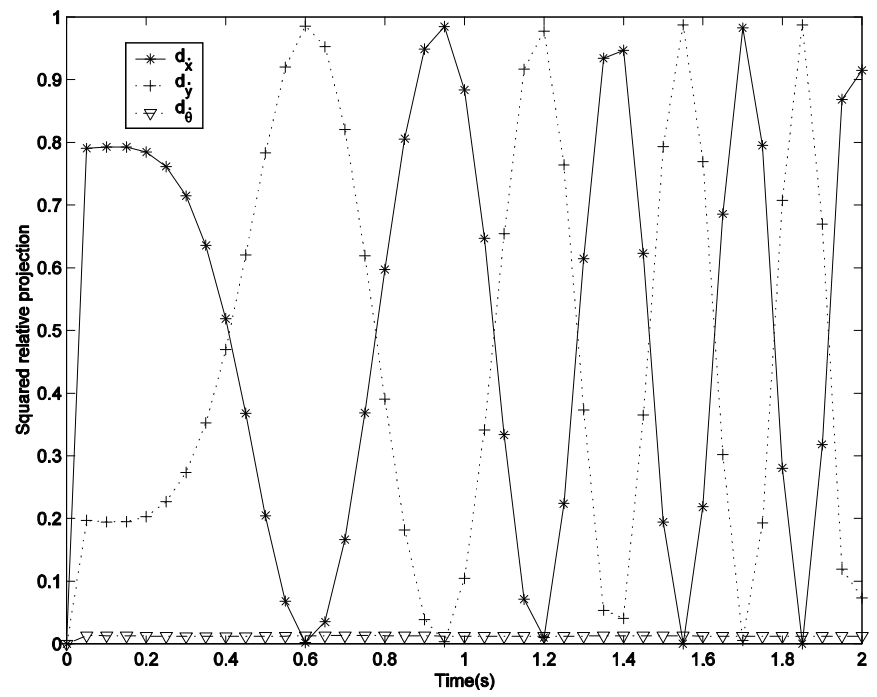

Figure 5: Evolution of the projections $d$

In figure 6 , the constraint violations errors are shown for the simulations without (S2) and with (S3) stabilisation of the constraint violation errors (29). For S3, the errors are theoretically zero (magnitude 1e-16 in practice, due to round off errors) the simulation. For the simulation without stabilisation, we see growing violation errors.

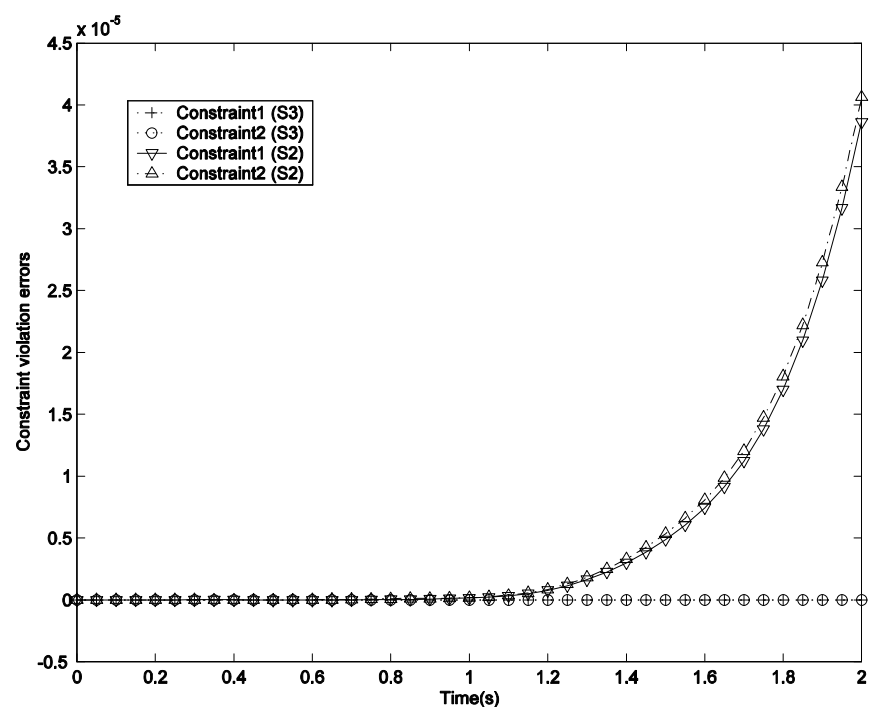

Figure 6: Constraint violation errors

During simulation $\mathrm{S} 4$, the second biggest projection (fig. 5) was used to choose the fourth independent velocity. Although this choice is valid and eliminates the constraint violation errors, it is not optimal and introduces larger errors along the trajectory, as shown in figure 7.

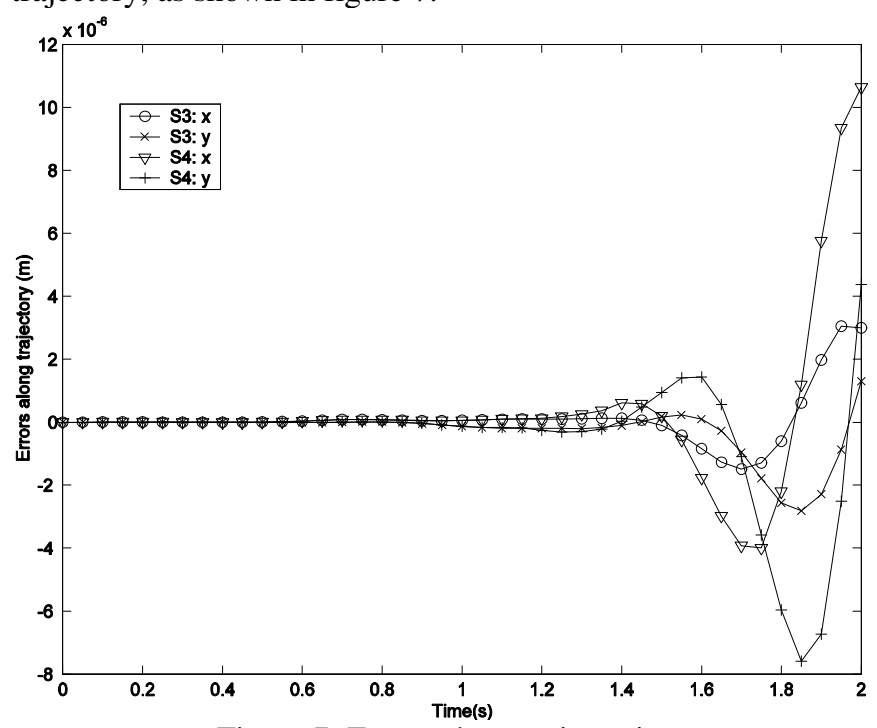

Figure 7: Errors along trajectories

\section{CONCLUSION}

The issues of geometric and stabilization characteristics of the constraint gradient projective method, which has been used as the stabilization procedure within time-integration method proposed in [5], have been addressed in the paper. By adopting differential-geometric point of view, the 'mechanism' of emerging of numerical errors along the 'position' configuration 
manifold during projection step have been discussed, along with the issue of stabilization of the constraints at the velocity level. In the case of simulation of holonomic systems, the optimal coordinate partitioning returns coordinate sub-vectors of the same structure at the both position and velocity level. Once procedure for position coordinates is completed, it must not be repeated for velocities.

It has been shown that constraint gradient projective method can be applied for stabilization of constraint violation of non-holonomic systems as well. Here, correction gradients to position and velocity sub-manifold do not match any more, which means that the optimal coordinates partitioning will not 'return' dependent/independent subvectors of the same structure for configuration and velocity stabilization. Generally, in the case of non-holonomic systems, the constraint gradient projective method should be performed separately for each stabilization level. This is specially true if the imposed nonholonomic constraints can not be put in Pfaffian form.

\section{REFERENCES}

[1] P. E. Nikravesh, Computer-Aided Analysis of Mechanical Systems, Prentice Hall (1988).

[2] W. Schiehlen, Multibody System Dynamics: Roots and Perspectives, Multibody System Dynamics, 1, 149-188 (1997).

[3] J. G. de Jalon, E. Bayo, Kinematic and Dynamic Simulation of Multibody Systems: The Real-Time Challenge, Springer-Verlag (1994).

[4] W. Blajer, Elimination of Constraint Violation and Accuracy Aspects in Numerical Simulation of Multibody Systems, Multibody System Dynamics, 7, 265-284 (2002).

[5] Z. Terze, D. Lefeber, O. Muftić, Null Space Integration Method for Constrained Multibody System Simulation with no Constraint Violation, Multibody System Dynamics, 6, 229-243 (2001).

[6] V. I. Arnold, Mathematical Methods of Classical Mechanics, Springer-Verlag, New York (1978).

[7] R. Abraham, J. E. Marsden, T. Ratiu, Manifolds, Tensor Analysis, and Applications, Springer-Verlag, New York (1988).

[8] R. Hermann, Constrained Mechanics and Lie theory, Math Sci Press, Brookline (1992).

[9] U. Jungnickel, Differential-Algebraic Equations in Riemannian Spaces and Applications to Multibody System Dynamics, Zeitschrift fuer Angewandte Mathematik und Mechanik, 74, 409-415 (1994).

[10] J. Simmonds, A Brief on Tensor Analysis, Springer Verlag, New York (1982).
[11] W. Blajer, A Geometric Unification of Constrained System Dynamics, Multibody System Dynamics, 1, 3-21 (1997).

[12] W. Schiehlen, Multibody Systems Handbook, Springer-Verlag, Berlin (1990).

[13] R. P. Singh and P. W. Likins, Singular Value Decomposition for Constrained Dynamical Systems, Journal of Applied Mechanics, 52, 943-948 (1985).

[14] S. K. Ider and F. M. L. Amirouche, Coordinate Reduction in the Dynamics of Constrained Multibody Systems-A New Approach, Journal of Applied Mechanics, 55, 899-904 (1988).

[15] W. Schiehlen, Technische Dynamik, B.G. Teubner, Stuttgart (1986).

[16] A. A. Shabana, Dynamics of Multibody Systems, Cambridge University Press (1998).

[17] F. E. Udwadia and R. E. Kalaba, The Geometry of Constrained Motion, Zeitschrift fuer Angewandte Mathematik und Mechanik, 75, 637-640 (1995).

[18] J. W. Kamman and R. L. Huston, Dynamics of Constrained Multibody Systems, Journal of Applied Mechanics, 51, 899-903 (1984).

[19] J. Cuadrado, J. Cardenal and E. Bayo, Modeling and Solution Methods for Efficient Real-Time Simulation of Multibody Dynamics, Multibody System Dynamics, 1, 259-280 (1997).

[20] R. A. Wehage, E. J. Haug, Generalized Coordinate Partitioning for Dimension Reduction in Analysis of Constrained Dynamic Systems, Journal of Mechanical Design, 104, 247-255 (1982).

[21] W. Blajer, W. Schiehlen and W. Schirm, A Projective Criterion to the Coordinate Partitioning Method for Multibody Dynamics, Archive of Applied Mechanics, 64, 86-98 (1994).

[22] Z. Terze, D Lefeber, Dynamic Simulation of Multibody Systems With no Constraints Violation, Transactions of FAMENA, 24, 1-9, (2000).

[23] S.G. Nash, A. Sofer, Linear and Nonlinear Programming, McGraw-Hill, New York (1996).

[24] J. V. Josè, E. J. Saletan, Classical Dynamics - A Contemporary Approach, Cambridge University Press, Camridge, UK (1998).

[25] A. Lewis, J. Ostrowski, R. Murray, J. Burdick, Nonholonomic Mechanics and Locomotion: The Snakeboard Example, IEEE Int. Conf. On Robotics and Automation, 23912397 (1994). 